\title{
Collective Neutrino Flavor Instability Requires a Crossing
}

\author{
Basudeb Dasgupta $\odot^{*}$ \\ Tata Institute of Fundamental Research, Homi Bhabha Road, Mumbai 400005, India
}

(Received 5 November 2021; revised 3 January 2022; accepted 10 February 2022; published 25 February 2022)

\begin{abstract}
Neutrinos in supernovae, neutron stars, and in the early Universe may change flavor collectively and unstably, due to neutrino-neutrino forward scattering. We prove that, for collective instability to occur, the difference of momentum distributions of two flavors must change sign, i.e., there is a zero crossing. This necessary criterion, which unifies slow and fast instabilities, is valid for Hamiltonian flavor evolution of ultrarelativistic standard model neutrino occupation matrices, including damping due to collisions in the relaxation approximation. It provides a simple but rigorous condition for collective flavor transformations that are believed to be important for stellar dynamics, nucleosynthesis, and neutrino phenomenology.
\end{abstract}

DOI: 10.1103/PhysRevLett.128.081102

Introduction.-Supernovae and neutron star mergers produce enormous numbers of neutrinos that carry away a bulk of the energy. These neutrinos travel through the dense material of the star, and crucially influence stellar dynamics and nucleosynthesis [1-6]. The precise impact can depend on the flavor states of the neutrinos, because the different flavors interact with the background medium with unequal interaction rates. Naturally, a characterization of the neutrino flavor evolution in such environments is of interest and importance.

The flavor evolution of dense neutrino clouds can be very complex [7-9]. Neutrinos produced in the core of these sources initially remain trapped due to frequent collisions. They leak out via diffusion, before eventually free-streaming. If the density of background matter is high, flavor mixing is suppressed due to forward and nonforward scatterings [10-12]. However, large collective flavor conversion can occur even for vanishingly small mixing. This novel instability arises when neutrinos forward scatter off each other and influence each other's flavor evolution. As a result, the flavor evolution becomes intricately coupled, i.e., collective, and creates nonlinear routes of exponentially growing flavor conversion.

In the last two decades, a lot of insight has been obtained into the origin and impact of a variety of collective flavor transformations. Collective flavor transformations stem from neutrino-neutrino forward scatterings [13,14], and come in three variants. The simplest type are synchronized, occurring for all neutrino energies with the average oscillation frequency $\left\langle\omega_{E}\right\rangle$, but not seeded by an

Published by the American Physical Society under the terms of the Creative Commons Attribution 4.0 International license. Further distribution of this work must maintain attribution to the author(s) and the published article's title, journal citation, and DOI. Funded by SCOAP ${ }^{3}$. instability [15]. These are usually suppressed in dense matter, though there is also the possibility of synchronized resonance that can cause large effects [16]. The next type are slow instabilities, leading to evolution with a frequency proportional to $\left(\left\langle\omega_{E}\right\rangle G_{F} n_{\nu}\right)^{1 / 2}$ [17-22], where $n_{\nu}$ is the neutrino density. At heart, these are analogous to the tipping of an inverted pendulum [22-24]. Despite being dubbed slow, they are faster than the usual neutrino oscillations in vacuum or matter, or even the synchronized oscillations, because $n_{\nu} \gg G_{F}^{-1}\left\langle\omega_{E}\right\rangle$ deep in the star. As the neutrino density drops below $G_{F}^{-1}\left\langle\omega_{E}\right\rangle$, typically at a radius of a few $\times 100 \mathrm{~km}$ in a supernova, these slow instabilities tend to produce a swap of two flavors across a broad range of energies $[23,24]$. The edges of these swaps could appear as sharp spectral splits in the energy spectrum and potentially observable in the signal reaching Earth. Finally, there are fast instabilities that cause flavor evolution with a very high frequency proportional to $G_{F} n_{\nu}$ [25-31]. These can occur very deep in a star at radii of few $\times 10 \mathrm{~km}$ or so [32-34], and may impact stellar heating and nucleosynthesis in a more nontrivial fashion. Fast instabilities correspond to, at their simplest, motion in a quartic potential [35] or tipping of a pendulum [36,37], and initially give wavelike propagation of flavor disturbance [38]. The eventual impact of these fast instabilities is not fully established yet, but a number of studies hint that they cause partial flavor equilibration in some range of neutrino velocities for all energies [39-42]. This is called depolarization, and may be a key observable of fast instability. The large flavor conversion encoded in spectral splits or depolarization affects neutrino transport, thus affecting stellar evolution $[43,44]$ and nucleosynthesis [45-47], in addition to giving unique signals at detectors [48].

A critical problem has been to determine the condition for collective instabilities. One belief has been that instabilities occur only if the flavor-difference distribution 
(FDD), i.e., the momentum distribution of the difference in the initial number densities of two neutrino flavors, changes sign at some momentum [24,28]. The importance of such FDD crossings was first pointed out in a study of multiple spectral splits [24]. Several subsequent investigations have further strengthened this notion for slow [49] and fast [30,50-52] instabilities. Recently, a proof was proposed for a necessary and sufficient condition for a fast instability [53].

In this Letter we show that "FDD crossings are necessary for collective instability." The argument is agnostic to whether the instability is slow or fast, to the number of neutrino flavors, and to whether damping due to collisions are present. This simple but rigorous criterion boxes in the astrophysical circumstances where collective neutrino flavor transformations may be important. In the following, we derive a linearized evolution equation including damping to prove the above claim, and then conclude with a brief summary and remarks.

Dispersion relation with damping.-We consider scenarios where neutrino flavor evolves as [54,55]

$$
v^{\alpha} \partial_{\alpha} \rho_{\mathbf{p}}=-i\left[\mathrm{H}_{\mathbf{p}}, \rho_{\mathbf{p}}\right]+\mathrm{C}_{\mathbf{p}},
$$

where $v^{\alpha}=(1, \mathbf{v})$ is the neutrino four velocity with $\mathbf{v}=\mathbf{p} /|E|$, and a summation over the spacetime indices $\alpha=0, \ldots, 3$ is implied. The $\rho_{\mathbf{p}}, \mathrm{H}_{\mathbf{p}}$, and $\mathrm{C}_{\mathbf{p}}$ are matrices in flavor space, with $\rho_{\mathbf{p}}$ (and $\bar{\rho}_{\mathbf{p}}$ ) encoding the occupation density and flavor coherence for neutrinos (and antineutrinos) in their diagonal and off-diagonal entries; $\mathrm{H}_{\mathbf{p}}$ and $\mathrm{C}_{\mathrm{p}}$ the Hamiltonian and collision matrices, respectively. The problem is nonlinear because $\mathrm{H}_{\mathbf{p}}$ contains terms involving $\rho_{\mathbf{p}}$ and $\bar{\rho}_{\mathbf{p}}$, as does $\mathbf{C}_{\mathbf{p}}$. The equation of motion (EOM) for $\bar{\rho}_{\mathbf{p}}$ is the same except for a sign change in the mass-mixing term in $\mathrm{H}_{\mathbf{p}}$.

In the following, we will derive a linearized equation for the off-diagonal elements $\rho_{\mathbf{p}}^{i j}$ in the flavor basis, where $i, j \in\{e, \mu, \tau\}$ in the usual three-flavor scenario. Our derivation remains essentially unchanged from Ref. [56], except for the inclusion of damping due to collisions.

The Hamiltonian matrix $H_{p}$ has the usual contributions from neutrino mass-mixing as well as the refractive effects of other neutrinos and background leptons:

$$
\mathrm{H}_{\mathbf{p}}=\frac{\mathrm{M}^{2}}{2 E}+\mathrm{H}_{\mathbf{p}}^{\nu \nu}+\mathrm{H}_{\mathbf{p}}^{\mathrm{bkg}} .
$$

Explicitly, the neutrino-neutrino refractive term has the form $\mathrm{H}_{\mathbf{p}}^{\nu \nu}=\sqrt{2} G_{F} v_{\alpha} \mathrm{F}_{\nu}^{\alpha}$ with the neutrino flux matrix $\mathrm{F}_{\nu}^{\alpha}=$ $\int đ \mathbf{p} v^{\alpha}\left(\rho_{\mathbf{p}}-\bar{\rho}_{\mathbf{p}}\right)$, where $₫ \mathbf{p}=d^{3} \mathbf{p} /(2 \pi)^{3}$. The ordinary matter contribution is $\mathrm{H}_{\mathbf{p}}^{\mathrm{bkg}}=\sqrt{2} G_{F} v_{\alpha} \mathrm{F}_{\mathrm{bkg}}^{\alpha}$, which is diagonal and has the elements $\left(\mathrm{F}_{\mathrm{bkg}}^{\alpha}\right)^{i i}=\int đ \mathbf{p} u_{i}^{\alpha}\left(f_{i, \mathbf{p}}-\bar{f}_{i, \mathbf{p}}\right)$, for the $i$ th charged lepton with phase space distribution $f_{i, \mathbf{p}}$ and a four-velocity $u_{i}^{\alpha}=\left[1, \mathbf{p} /\left(\mathbf{p}^{2}+m_{i}^{2}\right)^{1 / 2}\right]$. With only at-rest electrons in the background, one finds the familiar matter potential $\operatorname{diag}\left(2 \sqrt{2} G_{F} n_{e}, 0,0\right)$ for three flavors. The expression used here is more general and includes other (anti) leptons as well as their currents. The mass-mixing term does not depend on $\mathbf{v}$ and the refractive term does not depend on $E$, but only on $\mathbf{v}$. We may define an overall matter effect caused by both neutrinos and charged leptons as

$$
\mathrm{H}^{\text {matter,eff }}=v_{\alpha} \Lambda^{\alpha},
$$

where $\Lambda^{\alpha}=\operatorname{diag}\left(\Lambda_{e}^{\alpha}, \Lambda_{\mu}^{\alpha}, \Lambda_{\tau}^{\alpha}\right)$ to represent the diagonal part of $\sqrt{2} G_{F}\left(\mathrm{~F}_{\mathrm{bkg}}+\mathrm{F}_{\nu}\right)^{\alpha}$.

Collisions usually lead to damping of the off-diagonal flavor coherences [57-62]. This is because off-diagonal elements can be enhanced only when neutrinos are produced or scattered into flavor nondiagonal states. Chargedcurrent production or scattering leads to neutrinos in flavor eigenstates. Only neutral-current mediated scattering, with cross sections relatively suppressed by powers of $M_{W}^{2} / M_{Z}^{2}$, can lead to nondamping terms; e.g., a $Z$-mediated pairproduction (scattering) can give a flavor-mixed neutrino in the final state. We draw attention to the $d, c$, and $C$ terms in Sec. III of Ref. [55]; see also Refs. [63-67]. Nearly isotropic distributions will suppress the nondamping effects of scattering. In general, however, collisions can produce coherence that may mimic an instability.

We will restrict this work to include collisional processes in the relaxation approximation. For any pair of neutrino flavors, say $e$ and $\mu$, one has

$$
\mathrm{C}_{\mathbf{p}}^{e \mu}=-\left|\Delta_{\mathbf{p}}^{e \mu}\right| \rho_{\mathbf{p}}^{e \mu},
$$

with the damping rate $\left|\Delta_{\mathbf{p}}^{e \mu}\right|$ being non-negative. Similarly for any other pair of flavors.

In the limit of vanishing neutrino mixing, the linearized EoMs for the off-diagonal elements of $\rho_{\mathbf{p}}$ (and their complex conjugates) decouple, leading to equations of the form

$$
\begin{aligned}
i v^{\alpha} \partial_{\alpha} \rho_{\mathbf{p}}^{e \mu}= & \left(\frac{\mathrm{M}_{e e}^{2}-\mathbf{M}_{\mu \mu}^{2}}{2 E}-i\left|\Delta_{\mathbf{p}}^{e \mu}\right|+v_{\alpha}\left(\Lambda_{e}-\Lambda_{\mu}\right)^{\alpha}\right) \rho_{\mathbf{p}}^{e \mu} \\
& -\sqrt{2} G_{F}\left(f_{\nu_{e}, \mathbf{p}}-f_{\nu_{\mu}, \mathbf{p}}\right) v^{\alpha} \int đ \mathbf{p}^{\prime} v_{\alpha}^{\prime}\left(\rho_{\mathbf{p}^{\prime}}^{e \mu}-\bar{\rho}_{\mathbf{p}^{\prime}}^{e \mu}\right),
\end{aligned}
$$

and analogous for the other pairs of flavors.

In this approach the three-flavor system corresponds to three independent two-flavor cases. There are three nontrivial cases only if the distributions of the three flavors are different, as recently considered [68]. Extension to more than three flavors is obvious.

All flavor coherence effects depend only on the difference of the original neutrino distributions and the diagonal parts of all matrices in flavor space drop out. In particular, 
we may write the effective two-flavor neutrino matrices of occupation numbers in the form

$$
\varrho_{\mathbf{p}}^{e \mu}=\frac{f_{\nu_{e}, \mathbf{p}}+f_{\nu_{\mu}, \mathbf{p}}}{2} \mathbb{1}+\frac{f_{\nu_{e}, \mathbf{p}}-f_{\nu_{\mu}, \mathbf{p}}}{2}\left(\begin{array}{cc}
s_{\mathbf{p}} & S_{\mathbf{p}} \\
S_{\mathbf{p}}^{*} & -S_{\mathbf{p}}
\end{array}\right),
$$

whose off-diagonal element equals $\rho_{\mathbf{p}}^{e \mu}$, where $s_{\mathbf{p}}$ is a real number, $S_{\mathbf{p}}$ a complex one, and $s_{\mathbf{p}}^{2}+\left|S_{\mathbf{p}}\right|^{2}=1$. To linear order in $\left|S_{\mathbf{p}}\right|$, one has $s_{\mathbf{p}}=1$, so in our linearized study we focus on the space-time evolution of $S_{\mathbf{p}}$ alone which holds all the information concerning flavor coherence.

Defining the two-flavor matter effect through $\Lambda^{\alpha}=$ $\left(\Lambda_{e}-\Lambda_{\mu}\right)^{\alpha}$, the vacuum oscillation frequency through $\omega_{E}=\left(\mathrm{M}_{e e}^{2}-\mathrm{M}_{\mu \mu}^{2}\right) /(2 E)$, and the damping as $\left|\Delta_{\mathbf{p}}\right|$, the EOM in Eq. (5) becomes

$$
\begin{aligned}
i v^{\alpha} \partial_{\alpha} S_{\mathbf{p}}= & \left(\omega_{E}+v^{\alpha} \Lambda_{\alpha}-i\left|\Delta_{\mathbf{p}}\right|\right) S_{\mathbf{p}} \\
& -v^{\alpha} \int đ \mathbf{p}^{\prime} v_{\alpha}^{\prime}\left(S_{\mathbf{p}^{\prime}} g_{\mathbf{p}^{\prime}}-\bar{S}_{\mathbf{p}^{\prime}} \bar{g}_{\mathbf{p}^{\prime}}\right) .
\end{aligned}
$$

An analogous equation applies to the antineutrino flavor coherence $\bar{S}_{\mathbf{p}}$ with a sign-change of $\omega_{E}$. Here we use the FDD, $g_{\mathbf{p}}=\sqrt{2} G_{F}\left(f_{\nu_{e}, \mathbf{p}}-f_{\nu_{\mu}, \mathbf{p}}\right)$ and $\bar{g}_{\mathbf{p}}=\sqrt{2} G_{F}\left(f_{\bar{\nu}_{e}, \mathbf{p}}-f_{\bar{\nu}_{\mu}, \mathbf{p}}\right)$, where we have absorbed $\sqrt{2} G_{F}$ for notational convenience.

These equations become more compact and physically transparent in a convention where we interpret antiparticles as particles with negative energy and describe their FDD with negative occupation numbers. Thus the modes are labeled by $-\infty<E<+\infty$ and their direction of motion $\mathbf{v}$ with $\mathbf{p}=|E| \mathbf{v}$. The two-flavor FDD is

$$
g_{\Gamma}=\sqrt{2} G_{F} \begin{cases}f_{\nu_{e}, \mathbf{p}}-f_{\nu_{\mu}, \mathbf{p}} & \text { for } E>0, \\ f_{\bar{\nu}_{\mu}, \mathbf{p}}-f_{\bar{\nu}_{e}, \mathbf{p}} & \text { for } E<0,\end{cases}
$$

with $\Gamma=\{E, \mathbf{v}\}$. There is no sign change in the definition of $S$. The EOM thus reads

$\left[v^{\alpha}\left(i \partial_{\alpha}-\Lambda_{\alpha}\right)-\omega_{E}+i\left|\Delta_{\Gamma}\right|\right] S_{\Gamma}=-v^{\alpha} \int d \Gamma^{\prime} v_{\alpha}^{\prime} g_{\Gamma^{\prime}} S_{\Gamma^{\prime}}$,

where the phase-space integration is over

$$
\int d \Gamma=\int_{-\infty}^{+\infty} \frac{E^{2} d E}{2 \pi^{2}} \int \frac{d \mathbf{v}}{4 \pi}
$$

with $\int d \mathbf{v}$ an integral over the unit sphere, i.e., over the polar and azimuthal angles of $\mathbf{p}$.

The vacuum oscillation frequency $\omega_{E}$, in this convention, automatically changes sign for antineutrinos. For positive $E$, it is positive for inverted mass ordering $\left(\mathrm{M}_{e e}^{2}>\mathrm{M}_{\mu \mu}^{2}\right)$ and negative for the normal mass ordering $\left(\mathrm{M}_{e e}^{2}<\mathrm{M}_{\mu \mu}^{2}\right)$.
As usual, for a linear EOM we search for space-time dependent solutions of Eq. (9) in terms of its independent Fourier components

$$
S_{\Gamma, r}=\sum_{K} Q_{\Gamma, K} e^{-i\left(K_{0} t-\mathbf{K} \cdot \mathbf{r}\right)},
$$

where $r^{\mu}=(t, \mathbf{r})$ and $K^{\mu}=\left(K^{0}, \mathbf{K}\right)$. The quantity $Q_{\Gamma, K}$ is the eigenvector in $\Gamma$ space for the eigenvalue $K$. To find the eigenmodes we insert the ansatz of Eq. (11) into Eq. (9) and find

$$
\left(v_{\alpha} k^{\alpha}-\omega_{E}+i\left|\Delta_{\Gamma}\right|\right) Q_{\Gamma, k}=v_{\alpha} A_{k}^{\alpha},
$$

where $A_{k}^{\alpha}=-\int d \Gamma v^{\alpha} g_{\Gamma} Q_{\Gamma, k}$ and $k^{\alpha}=K^{\alpha}-\Lambda^{\alpha}$. Fully analogous to the fast-flavor case, we have shifted the original four-wave vector $K^{\mu}$ to the redefined four-wave vector, $k^{\mu}=\left(k^{0}, \mathbf{k}\right)$, by subtracting the matter-effect four vector $\Lambda^{\mu}$. Solving the EOM in Fourier space allows the diagonal parts of all matter effect to be included as an origin shift in the four-wave vector space.

In the absence of neutrino-neutrino interactions, the rhs of Eq. (12) vanishes and nontrivial solutions require $v_{\alpha} k^{\alpha}-\omega_{E}+i\left|\Delta_{\Gamma}\right|=0$, i.e., the propagation relation $\operatorname{Re} k_{0}-\mathbf{v} \cdot \mathbf{k}=\omega_{E}$ and the damping $\operatorname{Im} k_{0}=-\left|\Delta_{\Gamma}\right|$, where each neutrino mode labeled by $\{E, \mathbf{v}\}$ evolves independently. In the presence of neutrino-neutrino interactions, collective oscillations become possible where this dispersion relation changes. Therefore, we consider solutions with $v_{\alpha} k^{\alpha}-\omega_{E}+i\left|\Delta_{\Gamma}\right| \neq 0$ for any $\{E, \mathbf{v}\}$ so that Eq. (12) implies

$$
Q_{\Gamma, k}=\frac{v_{\alpha} A_{k}^{\alpha}}{v_{\gamma} k^{\gamma}-\omega_{E}+i\left|\Delta_{\Gamma}\right|} .
$$

Inserting this form on both sides of Eq. (12) yields

$$
v_{\alpha} A_{k}^{\alpha}=-v^{\alpha} A_{k}^{\beta} \int d \Gamma^{\prime} g_{\Gamma^{\prime}} \frac{v_{\alpha}^{\prime} v_{\beta}^{\prime}}{v_{\gamma}^{\prime} k^{\gamma}-\omega_{E^{\prime}}+i\left|\Delta_{\Gamma^{\prime}}\right|} .
$$

In more compact notation this can be written in the form

$$
v_{\alpha} \Pi_{k}^{\alpha \beta} A_{k, \beta}=0, \quad \text { where, }
$$

$$
\Pi_{k}^{\alpha \beta}=h^{\alpha \beta}+\int d \Gamma g_{\Gamma} \frac{v^{\alpha} v^{\beta}}{v_{\gamma} k^{\gamma}-\omega_{E}+i\left|\Delta_{\Gamma}\right|},
$$

with $h^{\alpha \beta}=\operatorname{diag}(+,-,-,-)$ being the metric tensor. This equation must hold for any $v^{\alpha}$ and thus consists of four independent equations $\Pi_{k}^{\alpha \beta} A_{k, \beta}=0$. Nontrivial solutions require

$$
\mathcal{D}(k) \equiv \operatorname{det} \Pi_{k}^{\alpha \beta}=0,
$$


establishing a connection between the components of $k=\left(k^{0}, \mathbf{k}\right)$, i.e., the dispersion relation of the system. It depends only on the neutrino FDD $g_{\Gamma}$, which itself contains the neutrino density, the vacuum oscillation frequency $\omega_{E}$, and the damping rate $\left|\Delta_{\Gamma}\right|$.

If the imaginary part of $k^{0}$ is positive, for any $k$ that satisfies Eqs. (17) and (11) tells us that it leads to exponential growth of the off-diagonal flavor coherence between the two flavors under consideration, i.e., $S^{e \mu} \sim e^{t \operatorname{Im} k^{0}}$. In the limit of vanishing flavor mixing, as relevant in dense matter, such flavor conversion is surprising and called a collective instability.

Proof that crossings are necessary.-Now we prove that collective instabilities can arise only if there is an FDD crossing. Technically, our proposition is that if any solution of the dispersion relation $\mathcal{D}(k)=0$ has $\operatorname{Im} k^{0} \equiv \sigma>0$ and $\mathbf{k} \in \mathbb{R}^{3}$, then the FDD (i.e., $g_{\Gamma}$ ) cannot have the same sign everywhere. We will prove the proposition by contradiction, following Morinaga [53], but cover a potential singular case.

In the following, we omit explicitly noting the $k$ dependence of the matrix $\Pi_{k}$ and its eigenvector $A_{k}$. Also we separate the real and imaginary parts of $k^{0}=\kappa+i \sigma$, where $\kappa, \sigma \in \mathbb{R}$, and write the $\Pi$ matrix as

$$
\Pi^{\alpha \beta}=M^{\alpha \beta}-i N^{\alpha \beta},
$$

where $M$ and $N$ are real-symmetric matrices

$$
\begin{aligned}
M^{\alpha \beta} & =h^{\alpha \beta}+\int d \Gamma g_{\Gamma} \frac{\left(\kappa-\mathbf{v} \cdot \mathbf{k}-\omega_{E}\right) v^{\alpha} v^{\beta}}{\left(\kappa-\mathbf{v} \cdot \mathbf{k}-\omega_{E}\right)^{2}+\left(\sigma+\left|\Delta_{\Gamma}\right|\right)^{2}}, \\
N^{\alpha \beta} & =\int d \Gamma g_{\Gamma} \frac{\left(\sigma+\left|\Delta_{\Gamma}\right|\right) v^{\alpha} v^{\beta}}{\left(\kappa-\mathbf{v} \cdot \mathbf{k}-\omega_{E}\right)^{2}+\left(\sigma+\left|\Delta_{\Gamma}\right|\right)^{2}} .
\end{aligned}
$$

The matrix $N$ can be diagonalized by a real orthogonal matrix $O$ as

$$
O_{\mu}^{\alpha} O_{\nu}^{\beta} N^{\mu \nu}=D^{\alpha \beta},
$$

where $D$ is a diagonal matrix whose components are

$$
D^{\alpha \alpha}=\int d \Gamma g_{\Gamma} \frac{\left(\sigma+\left|\Delta_{\Gamma}\right|\right)\left(O_{\mu}^{\alpha} v^{\mu}\right)^{2}}{\left(\kappa-\mathbf{v} \cdot \mathbf{k}-\omega_{E}\right)^{2}+\left(\sigma+\left|\Delta_{\Gamma}\right|\right)^{2}} .
$$

In this basis where $N$ becomes diagonal, the matrix $M$ becomes $\tilde{M}$ and the dispersion relation $\mathcal{D}(k)=0$ becomes $\operatorname{det}(\tilde{M}-i D)=0$, which implies that there exists a nontrivial four-eigenvector $A$ such that

$$
\tilde{M}^{\alpha \beta} A_{\beta}=+i D^{\alpha \beta} A_{\beta} .
$$

Note that $\tilde{M}-i D$ is a complex-symmetric matrix, so, in general, $A$ is a complex vector. We multiply the above equation by $A_{\alpha}^{*}$ and sum over $\alpha$ to get

$$
\tilde{M}^{\alpha \beta} A_{\alpha}^{*} A_{\beta}=+i D^{\alpha \beta} A_{\alpha}^{*} A_{\beta},
$$

whose complex conjugate is given by

$$
\tilde{M}^{\alpha \beta} A_{\alpha} A_{\beta}^{*}=-i D^{\alpha \beta} A_{\alpha} A_{\beta}^{*} .
$$

Using the fact that $\alpha$ and $\beta$ are dummy indices and can be renamed $\beta$ and $\alpha$, respectively, and that $\tilde{M}$ is symmetric, i.e., $\tilde{M}^{\beta \alpha}=\tilde{M}^{\alpha \beta}$, we get

$$
\tilde{M}^{\alpha \beta} A_{\alpha}^{*} A_{\beta}=-i D^{\alpha \beta} A_{\alpha}^{*} A_{\beta} .
$$

Subtracting Eq. (25) from Eq. (23) gives

$$
\sum_{\alpha} D^{\alpha \alpha}\left|A_{\alpha}\right|^{2}=0
$$

In Eq. (26) above, $\left|A_{\alpha}\right|^{2}$ are non-negative and not all of them vanish. As proposed, we have $\sigma>0$ and $g_{\Gamma}$ has the same sign everywhere, so Eq. (21) dictates that all $D^{\alpha \alpha}$ have the same signature as $g_{\Gamma}$.

There would appear to be two possibilities for Eq. (26). First, the singular case where $D^{\alpha \alpha}=0$ for all $\alpha$ for which $\left|A_{\alpha}\right|^{2} \neq 0$. However, in this case Eq. (21) requires that for those $\alpha$, the integral of $\left(O_{\mu}^{\alpha} v^{\mu}\right)^{2}$ times an everywheresame-sign function vanishes identically. This is possible only if $\left(O_{\mu}^{\alpha} v^{\mu}\right)^{2}=0$ for all points in $\Gamma$ or if $g_{\Gamma}=0$. That is, the same $O$ makes the $\alpha$ component of any $v$ vanish or that there are no collective effects at all, respectively. These are either impossible or trivial, and therefore excluded. Second is the nonsingular case, where $D^{\alpha \alpha} \neq 0$ for some $A_{\alpha} \neq 0$. In this case, in Eq. (26) at least one term is nonzero and all terms are of the same sign. But then Eq. (26), which algebraically followed from our original assumptions, cannot be satisfied. The only resolution is that $g_{\Gamma}$ must change sign if there exists a $\sigma>0$. This completes the proof of the proposition. As a corollary, setting $\omega_{E} \rightarrow 0$ and $\Delta_{\Gamma} \rightarrow 0$, one recovers the necessary condition for collisionless fast instability [53].

Discussion and outlook.-We have shown that collective flavor instability requires an FDD crossing in momentum space. Observation of signatures of collective neutrino flavor instabilities, such as spectral splits, depolarization, and their impact on stellar heating and nucleosynthesis, will therefore provide information on neutrino distributions deep inside stars. The criterion presented in this work gives a rigorous foundation for this physical expectation. It also makes it eminently sensible to rule out collective flavor instabilities in supernova simulations by simply ruling out FDD crossings [69-71]. We note that this criterion unifies the origin of slow and fast instabilities, and is distinct from the crossing criterion for fast instabilities where one demands a crossing in the energy-integrated FDD [53]. Also, note that the proof does not depend on any detailed 
features of $g_{\Gamma}$; thus it cannot be violated by invoking azimuthally non-symmetric distributions, for example.

We now outline some limitations of the necessary criterion. Our framework considers evolution of the occupation matrices, $\rho \sim\left\langle a^{\dagger} a\right\rangle$ and $\bar{\rho} \sim\left\langle b^{\dagger} b\right\rangle$, related to fieldtheoretic expectations of the fermion bilinears. Other correlations, e.g., $\left\langle a^{\dagger} b\right\rangle$, encode coupling of helicity, spin, lepton-number. While much tinier, these demand an expanded Hilbert space [63-67], which could invalidate the proof. Similarly, going beyond the mean-field approximation [72,73] needs a more sophisticated treatment. Beyond the Standard Model, even certain kinds of forward interactions render the linearized EOM to not remain an eigenvalue equation [74], and the proof ceases to apply. Also, note that our analysis is limited to linear instability. Finally, within the assumed framework, the collision term could have nondamping parts that we have ignored. These extensions may reveal novel collective oscillation effects that are not accounted for by the crossing criterion that was proved here.

One could ask if an FDD crossing is also sufficient for a collective instability? In the fast limit, it has been proposed that a crossing in the energy-integrated FDD spectrum must lead to some solution $k$ of the dispersion relation $\mathcal{D}(k)=0$ with a complex $k^{0}$ and real $\mathbf{k}$ [53]. The proof in effect recasts the dispersion relation as a quartic polynomial, which we could not reproduce. Note also, even if a crossing guarantees instability, the rate of the instability can be very small.

The exploration of collective effects over the past three decades has revealed many novel phenomena. The proposed necessary criterion hopefully provides an organizing principle, and its violations may reveal yet newer secrets of collective neutrino flavor transformations.

It is my pleasure to thank S. Abbar, T. Morinaga, G. Raffelt, and R. Sawyer for detailed discussions. I also thank A. Dighe, L. Johns, and M. Sen for helpful exchanges. This work is supported by the Department of Atomic Energy (Government of India) research project RTI 4002, the Department of Science and Technology (Government of India) through a Swarnajayanti Fellowship, and by the Max-Planck-Gesellschaft through a Max Planck Partner Group.

*bdasgupta@theory.tifr.res.in

[1] S. A. Colgate and R. H. White, The hydrodynamic behavior of supernovae explosions, Astrophys. J. 143, 626 (1966).

[2] H. A. Bethe, Supernova mechanisms, Rev. Mod. Phys. 62, 801 (1990).

[3] H. T. Janka, T. Melson, and A. Summa, Physics of corecollapse supernovae in three dimensions: A sneak preview, Annu. Rev. Nucl. Part. Sci. 66, 341 (2016).

[4] A. Burrows and D. Vartanyan, Core-collapse supernova explosion theory, Nature (London) 589, 29 (2021).
[5] F. K. Thielemann, M. Eichler, I. V. Panov, and B. Wehmeyer, Neutron star mergers and nucleosynthesis of heavy elements, Annu. Rev. Nucl. Part. Sci. 67, 253 (2017).

[6] J. J. Cowan, C. Sneden, J. E. Lawler, A. Aprahamian, M. Wiescher, K. Langanke, G. Martinez-Pinedo, and F. K. Thielemann, Origin of the heaviest elements: The rapid neutron-capture process, Rev. Mod. Phys. 93, 015002 (2021).

[7] H. Duan, G. M. Fuller, and Y.-Z. Qian, Collective neutrino oscillations, Annu. Rev. Nucl. Part. Sci. 60, 569 (2010).

[8] S. Chakraborty, R. Hansen, I. Izaguirre, and G. Raffelt, Collective neutrino flavor conversion: Recent developments, Nucl. Phys. B908, 366 (2016).

[9] I. Tamborra and S. Shalgar, New developments in flavor evolution of a dense neutrino gas, Annu. Rev. Nucl. Part. Sci. 71, 165 (2021).

[10] L. Wolfenstein, Neutrino oscillations in matter, Phys. Rev. D 17, 2369 (1978).

[11] S. P. Mikheev and A. Yu. Smirnov, Neutrino oscillations in an inhomogeneous medium: Adiabatic regime, Sov. Phys. JETP 65, 230 (1987).

[12] L. Stodolsky, On the treatment of neutrino oscillations in a thermal environment, Phys. Rev. D 36, 2273 (1987).

[13] J. T. Pantaleone, Dirac neutrinos in dense matter, Phys. Rev. D 46, 510 (1992).

[14] J. T. Pantaleone, Neutrino oscillations at high densities, Phys. Lett. B 287, 128 (1992).

[15] V. A. Kostelecky and S. Samuel, Selfmaintained coherent oscillations in dense neutrino gases, Phys. Rev. D 52, 621 (1995).

[16] Y.Z. Qian and G. M. Fuller, Neutrino-neutrino scattering and matter enhanced neutrino flavor transformation in Supernovae, Phys. Rev. D 51, 1479 (1995).

[17] V. A. Kostelecky and S. Samuel, Neutrino oscillations in the early universe with an inverted neutrino mass hierarchy, Phys. Lett. B 318, 127 (1993).

[18] S. Pastor, G. G. Raffelt, and D. V. Semikoz, Physics of synchronized neutrino oscillations caused by selfinteractions, Phys. Rev. D 65, 053011 (2002).

[19] A. Friedland and C. Lunardini, Neutrino flavor conversion in a neutrino background: Single particle versus multiparticle description, Phys. Rev. D 68, 013007 (2003).

[20] H. Duan, G. M. Fuller, and Y.-Z. Qian, Collective neutrino flavor transformation in supernovae, Phys. Rev. D 74, 123004 (2006).

[21] H. Duan, G. M. Fuller, J. Carlson, and Y.-Z. Qian, Simulation of coherent non-linear neutrino flavor transformation in the supernova environment. 1. Correlated neutrino trajectories, Phys. Rev. D 74, 105014 (2006).

[22] S. Hannestad, G. G. Raffelt, G. Sigl, and Y. Y. Wong, Selfinduced conversion in dense neutrino gases: Pendulum in flavour space, Phys. Rev. D 74, 105010 (2006).

[23] G. G. Raffelt and A. Y. Smirnov, Self-induced spectral splits in supernova neutrino fluxes, Phys. Rev. D 76, 081301(R) (2007).

[24] B. Dasgupta, A. Dighe, G. G. Raffelt, and A. Y. Smirnov, Multiple Spectral Splits of Supernova Neutrinos, Phys. Rev. Lett. 103, 051105 (2009).

[25] R. Sawyer, Speed-up of neutrino transformations in a supernova environment, Phys. Rev. D 72, 045003 (2005). 
[26] R. F. Sawyer, Neutrino Cloud Instabilities Just Above the Neutrino Sphere of a Supernova, Phys. Rev. Lett. 116, 081101 (2016).

[27] S. Chakraborty, R. S. Hansen, I. Izaguirre, and G. Raffelt, Self-induced neutrino flavor conversion without flavor mixing, J. Cosmol. Astropart. Phys. 03 (2016) 042.

[28] B. Dasgupta, A. Mirizzi, and M. Sen, Fast neutrino flavor conversions near the supernova core with realistic flavordependent angular distributions, J. Cosmol. Astropart. Phys. 02 (2017) 019.

[29] I. Izaguirre, G. Raffelt, and I. Tamborra, Fast Pairwise Conversion of Supernova Neutrinos: A Dispersion-Relation Approach, Phys. Rev. Lett. 118, 021101 (2017).

[30] F. Capozzi, B. Dasgupta, E. Lisi, A. Marrone, and A. Mirizzi, Fast flavor conversions of supernova neutrinos: Classifying instabilities via dispersion relations, Phys. Rev. D 96, 043016 (2017).

[31] C. Yi, L. Ma, J. D. Martin, and H. Duan, Dispersion relation of the fast neutrino oscillation wave, Phys. Rev. D 99, 063005 (2019).

[32] T. Morinaga, H. Nagakura, C. Kato, and S. Yamada, Fast neutrino-flavor conversion in the preshock region of corecollapse supernovae, Phys. Rev. Research 2, 012046(R) (2020).

[33] S. Abbar, H. Duan, K. Sumiyoshi, T. Takiwaki, and M. C. Volpe, Fast neutrino flavor conversion modes in multidimensional core-collapse supernova models: The role of the asymmetric neutrino distributions, Phys. Rev. D 101, 043016 (2020).

[34] R. Glas, H. T. Janka, F. Capozzi, M. Sen, B. Dasgupta, A. Mirizzi, and G. Sigl, Fast neutrino flavor instability in the neutron-star convection layer of three-dimensional supernova models, Phys. Rev. D 101, 063001 (2020).

[35] B. Dasgupta and M. Sen, Fast neutrino flavor conversion as oscillations in a quartic potential, Phys. Rev. D 97, 023017 (2018).

[36] L. Johns, H. Nagakura, G. M. Fuller, and A. Burrows, Neutrino oscillations in supernovae: Angular moments and fast instabilities, Phys. Rev. D 101, 043009 (2020).

[37] S. Bhattacharyya and B. Dasgupta, Late-time behavior of fast neutrino oscillations, Phys. Rev. D 102, 063018 (2020).

[38] J. D. Martin, C. Yi, and H. Duan, Dynamic fast flavor oscillation waves in dense neutrino gases, Phys. Lett. B 800, 135088 (2020).

[39] S. Bhattacharyya and B. Dasgupta, Fast Flavor Depolarization of Supernova Neutrinos, Phys. Rev. Lett. 126, 061302 (2021).

[40] L. Johns, H. Nagakura, G. M. Fuller, and A. Burrows, Fast oscillations, collisionless relaxation, and spurious evolution of supernova neutrino flavor, Phys. Rev. D 102, 103017 (2020).

[41] M.-R. Wu, M. George, C.-Y. Lin, and Z. Xiong, Collective fast neutrino flavor conversions in an 1D box: (I) initial condition and long-term evolution, Phys. Rev. D 104, 103003 (2021).

[42] S. Richers, D. E. Willcox, N. M. Ford, and A. Myers, Particle-in-cell simulation of the neutrino fast flavor instability, Phys. Rev. D 103, 083013 (2021).

[43] O. Pejcha, B. Dasgupta, and T. A. Thompson, Effect of collective neutrino oscillations on the neutrino mechanism of core-collapse supernovae, Mon. Not. R. Astron. Soc. 425, 1083 (2012).

[44] B. Dasgupta, E. P. O'Connor, and C. D. Ott, The role of collective neutrino flavor oscillations in core-collapse supernova shock revival, Phys. Rev. D 85, 065008 (2012).

[45] H. Duan, A. Friedland, G. McLaughlin, and R. Surman, The influence of collective neutrino oscillations on a supernova r-process, J. Phys. G 38, 035201 (2011).

[46] Z. Xiong, A. Sieverding, M. Sen, and Y.-Z. Qian, Potential impact of fast flavor oscillations on neutrino-driven winds and their nucleosynthesis, Astrophys. J. 900, 144 (2020).

[47] X. Li and D. M. Siegel, Neutrino Fast Flavor Conversions in Neutron-Star Postmerger Accretion Disks, Phys. Rev. Lett. 126, 251101 (2021).

[48] F. Capozzi, B. Dasgupta, and A. Mirizzi, Model-independent diagnostic of self-induced spectral equalization versus ordinary matter effects in supernova neutrinos, Phys. Rev. D 98, 063013 (2018).

[49] A. Banerjee, A. Dighe, and G. Raffelt, Linearized flavorstability analysis of dense neutrino streams, Phys. Rev. D 84, 053013 (2011).

[50] S. Abbar and H. Duan, Fast neutrino flavor conversion: Roles of dense matter and spectrum crossing, Phys. Rev. D 98, 043014 (2018).

[51] F. Capozzi, G. Raffelt, and T. Stirner, Fast neutrino flavor conversion: Collective motion vs decoherence, J. Cosmol. Astropart. Phys. 09 (2019) 002.

[52] T. Morinaga, Spatiotemporal linear instability analysis of collective neutrino flavor conversion in four-dimensional spacetime, Phys. Rev. D 103, 083014 (2021).

[53] T. Morinaga, Fast neutrino flavor instability and neutrino flavor lepton number crossings, arXiv:2103.15267.

[54] G. Sigl and G. Raffelt, General kinetic description of relativistic mixed neutrinos, Nucl. Phys. B406, 423 (1993).

[55] B. H. J. McKellar and M. J. Thomson, Oscillating doublet neutrinos in the early universe, Phys. Rev. D 49, 2710 (1994).

[56] S. Airen, F. Capozzi, S. Chakraborty, B. Dasgupta, G. Raffelt, and T. Stirner, Normal-mode analysis for collective neutrino oscillations, J. Cosmol. Astropart. Phys. 12 (2018) 019.

[57] M. Sen, New aspects of supernova neutrino flavor conversions: In the standard model and beyond, Ph.D. thesis, Tata Institute of Fundamental Research, 8, 2018.

[58] F. Capozzi, B. Dasgupta, A. Mirizzi, M. Sen, and G. Sigl, Collisional Triggering of Fast Flavor Conversions of Supernova Neutrinos, Phys. Rev. Lett. 122, 091101 (2019).

[59] S. Shalgar and I. Tamborra, A change of direction in pairwise neutrino conversion physics: The effect of collisions, Phys. Rev. D 103, 063002 (2021).

[60] J. D. Martin, J. Carlson, V. Cirigliano, and H. Duan, Fast flavor oscillations in dense neutrino media with collisions, Phys. Rev. D 103, 063001 (2021).

[61] L. Johns, Collisional flavor instabilities of supernova neutrinos, arXiv:2104.11369.

[62] H. Sasaki and T. Takiwaki, Dynamics of fast neutrino flavor conversions with scattering effects: A detailed analysis, arXiv:2109.14011.

[63] C. Volpe, D. Väänänen, and C. Espinoza, Extended evolution equations for neutrino propagation in astrophysical and cosmological environments, Phys. Rev. D 87, 113010 (2013). 
[64] A. Vlasenko, G. M. Fuller, and V. Cirigliano, Neutrino quantum kinetics, Phys. Rev. D 89, 105004 (2014).

[65] A. Kartavtsev, G. Raffelt, and H. Vogel, Neutrino propagation in media: Flavor-, helicity-, and pair correlations, Phys. Rev. D 91, 125020 (2015).

[66] D. N. Blaschke and V. Cirigliano, Neutrino quantum kinetic equations: The collision term, Phys. Rev. D 94, 033009 (2016).

[67] S. A. Richers, G. C. McLaughlin, J.P. Kneller, and A. Vlasenko, Neutrino quantum kinetics in compact objects, Phys. Rev. D 99, 123014 (2019).

[68] F. Capozzi, M. Chakraborty, S. Chakraborty, and M. Sen, Fast Flavor Conversions in Supernovae: The Rise of $\mathrm{Mu}-$ Tau Neutrinos, Phys. Rev. Lett. 125, 251801 (2020).

[69] B. Dasgupta, A. Mirizzi, and M. Sen, Simple method of diagnosing fast flavor conversions of supernova neutrinos, Phys. Rev. D 98, 103001 (2018).
[70] S. Abbar, Searching for fast neutrino flavor conversion modes in core-collapse supernova simulations, J. Cosmol. Astropart. Phys. 05 (2020) 027.

[71] L. Johns and H. Nagakura, Fast flavor instabilities and the search for neutrino angular crossings, Phys. Rev. D 103, 123012 (2021).

[72] A. V. Patwardhan, M. J. Cervia, and A. Baha Balantekin, Eigenvalues and eigenstates of the many-body collective neutrino oscillation problem, Phys. Rev. D 99, 123013 (2019).

[73] A. Roggero, Entanglement and many-body effects in collective neutrino oscillations, Phys. Rev. D 104, 103016 (2021).

[74] A. Dighe and M. Sen, Nonstandard neutrino selfinteractions in a supernova and fast flavor conversions, Phys. Rev. D 97, 043011 (2018). 